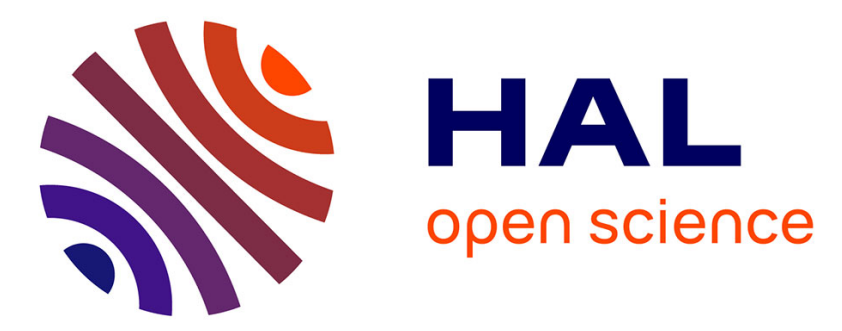

\title{
Optimization of Magnet Segmentation for Reduction of Eddy-Current Losses in Permanent Magnet Synchronous Machine
}

Wan-Ying Huang, Adel Bettayeb, Robert Kaczmarek, Jean-Claude Vannier

\section{- To cite this version:}

Wan-Ying Huang, Adel Bettayeb, Robert Kaczmarek, Jean-Claude Vannier. Optimization of Magnet Segmentation for Reduction of Eddy-Current Losses in Permanent Magnet Synchronous Machine. IEEE Transactions on Energy Conversion, 2010, 25 (2), pp.381-387. hal-00491390

\section{HAL Id: hal-00491390 \\ https://hal-centralesupelec.archives-ouvertes.fr/hal-00491390}

Submitted on 15 Jun 2010

HAL is a multi-disciplinary open access archive for the deposit and dissemination of scientific research documents, whether they are published or not. The documents may come from teaching and research institutions in France or abroad, or from public or private research centers.
L'archive ouverte pluridisciplinaire HAL, est destinée au dépôt et à la diffusion de documents scientifiques de niveau recherche, publiés ou non, émanant des établissements d'enseignement et de recherche français ou étrangers, des laboratoires publics ou privés. 


\title{
Optimization of Magnet Segmentation for Reduction of Eddy-Current Losses in Permanent Magnet Synchronous Machine
}

\author{
Wan-Ying Huang, Adel Bettayeb, Robert Kaczmarek, and Jean-Claude Vannier
}

\begin{abstract}
Rare Earth Permanent magnet (PM) synchronous machines with the pulse width modulated (PWM) supply are exposed to magnets' heating due to harmonic fields which rotate in relation to rotor. In cases when skin effect is negligible, the corresponding eddy-current losses in magnets can be reduced by segmentation of magnets. In this paper we propose an analytical method to evaluate the effect of both circumferential and axial segmentations on losses' reduction in various conditions concerning the skin effect. With the skin effect operating, the magnets' division may lead to increase rather than decrease of the eddy-current losses. A criterion to determine range of parameters leading to this unexpected situation is presented. Finite element calculation confirms the results of the analysis.
\end{abstract}

Index Terms - Eddy-currents, power losses, skin effect. Permanent magnet machines, finite-element methods.

\section{INTRODUCTION}

$\mathrm{W}$ HEN designing high-speed permanent magnet (PM) machine, an effort to obtain eddy-current losses reduction in the magnets is necessary in order to improve their performances and diminish the risk of demagnetization.

Circumferential segmentation [1]-[3] of PM has been considered as efficient methods of reduction of the eddycurrent losses, in cases when pole-arc width and radial dimension of magnet segments are less than the skin depth of interest. Axial segmentation, initially considered inefficient [2], has also been proved beneficial for reduction of losses [4], [5].

An analytical equation of the eddy-current losses in magnets, due to time harmonics in stator currents, has been developed [1] to account for effect of PM circumferential

Manuscript received June 10, 2009.

W-Y. Huang is with the Department of Electrical Machines \& Power Systems of Supélec, $91192 \quad$ Gif-sur-Yvette, France (wanying.huang@yahoo.fr)

A. Bettayeb is with the Department of Electrical Machines \& Power Systems of Supélec, 91192 Gif-sur-Yvette, France (adel.bettayeb@supelec.fr)

R. Kaczmarek is with the Department of Electrical Machines \& Power Systems of Supélec, 91192 Gif-sur-Yvette, France (robert.kaczmarek@supelec.fr)

J-C. Vannier is with the Department of Electrical Machines \& Power Systems of Supélec, 91192 Gif-sur-Yvette, France (jeanclaude.vannier@supelec.fr) segmentations. This equation was based on assumption that the pole-arc dimension of magnet is so small that flux density over it is uniform. Performance of this equation has been proved by comparing calculated and measured impedances of PM machine for different harmonics at $1 \sim 100 \mathrm{kHz}$.

In [2-4], analytical method for predicting the eddy-current losses in magnets, due to space harmonics of magneto motive force (MMF), has been presented and used to quantify the efficiency of one dimensional (circumferential or axial) segmentations of PM when looking for loss reduction. The reported analytical techniques to evaluate the eddy-current losses, for the cases when skin depth at the frequencies of interest is greater than both pole-arc and radial dimensions of magnets, were validated by 2-D time-stepping finite element (FE) analysis [2-3], and by 3-D magneto-static FE analysis [4].

However, the condition that "skin depth is greater than both pole-arc width and radial height", is not always satisfied. Out of the concerned range of parameters, PM segmentation may increase rather than reduce the eddy-current loss. We will call the unexpected deviation of this rule the "anomaly of segmentation".

Recently, the anomaly with respect to axial segmentation has been shown in [5] for Interior PM motor, using 3-D timestepping finite element nonlinear analysis with very important calculating time. To explain these results, authors introduced complicate theoretical solutions of the eddy-current losses in thin conductor, when the uniform magnetic field is applied. When the skin depth $\delta$ is large enough in relation to the width $w$ and the length $l$ of magnet segments, an approximation was made to obtain a simpler equation of losses, which represents the reduction of losses with respect to axial magnet divisions. However, we estimate that it's still rather complicated and we need numerical calculation to comprehend the effect of magnet segmentations.

On the other hand, when $\delta$ is much smaller than $w$ and $l$, another approximation was given to represent the increase of losses with respect to magnet divisions. By observing the results, authors determine that the eddy-current losses become maximum when the length of conductor is nearly twice of the skin depth.

In our paper, a surface-mounted PM synchronous machine with 72 stator slots fed by pulse width modulated (PWM) voltage supply is designed with quasi sinusoidal winding 
distribution. The harmonics analysis is concerned with the time harmonics of order 5 and 7,11 and 13, 17 and 19 etc. The magnets' surface per pole has dimensions $70 \mathrm{~mm} * 250 \mathrm{~mm}$.

In case of non-sinusoidal supply the eddy-current losses cannot be given by scalar summation of separately predicted loses for each harmonic. As a matter of fact, the "relevant harmonics", e.g. the $5^{\text {th }}$ and the $7^{\text {th }}$ (or the $11^{\text {th }}$ and the $13^{\text {th }}$ ) time harmonics induce in rotor the eddy-currents with the same $6^{\text {th }}$ (or $12^{\text {th }}$ ) harmonic frequency but in opposite directions $(-6 \omega$ and $+6 \omega)$. Superposition of individual loss may differ substantially from losses dissipated by composed fields [6].

Instead of superposition of losses separately resulting from the two relevant harmonics, in this paper we will firstly calculate the composed flux density and then evaluate the corresponding losses.

The aim of this paper is to determine by simple criterion the range of parameters within which the anomaly of PM segmentation can take place. In other words, only out of this range we can try to optimize the PM segmentation in both the circumferential and axial directions. The analytical predictions of the eddy-current losses with effect of magnet segmentations are validated by 3-D FE harmonic analysis with locked rotor technique, taking about $1 \mathrm{~h}$ for $\mathrm{PC}$ calculation, the time amount largely inferior when compared to that reported with time-stepping method.

\section{EdDy-CurRent Losses IN CASE OF NEGLIGIBLE SKIN EFFECT}

\section{A. Traditional Analytical Model}

An analytical model (1) of the eddy-current loss per unit volume $\mathrm{P}_{\mathrm{v}}$ [1], [7]-[9], often applied to laminated steel, can be used to explain the idea of PM pole-arc segmentation for reducing eddy-current loss.

$P_{v}=\frac{\pi^{2} f^{2} B_{m}^{2} w^{2}}{6 \rho}$

where $\rho$ is PM resistivity, $w$ is pole-arc width of PM segmentation, $B_{m}$ is maximum instantaneous flux density in magnets and $f$ is its frequency. It shows that the eddy-current losses per unit volume are proportional to square of the width $w$. The losses diminish when the magnets are divided into smaller segments.

In this equation, following assumptions are used:

1) The width $w$ is less than the skin depth so that the skin effect can be neglected.

2) The magnetic flux density $B$ is homogeneous over the PM width.

3) The width $w$ is much smaller than the PM segmentation length $l$, so that end effect can be neglected.

However, all these three assumptions may be controversial in general cases. For example, the widths of magnet segments between $10 \mathrm{~mm}$ and $70 \mathrm{~mm}$ may be greater than the skin depth

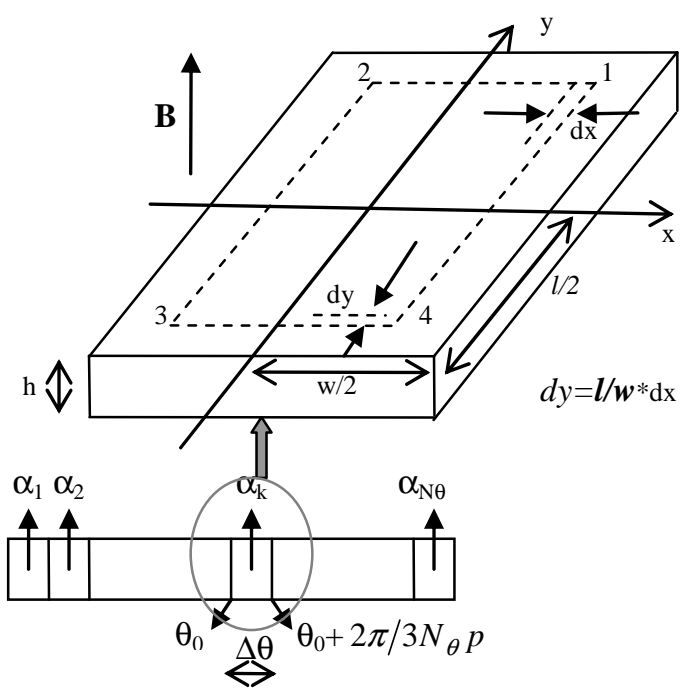

Fig. 1. Cross section of one pole magnet and one of its segmentation

of interest, and the flux density resulting from stator currents can't be considered homogeneous over a large width of magnet (e.g. $70 \mathrm{~mm}$, covering about $1 / 3$ of one pole), even if the skin effect could be neglected. In fact, the flux density is sum of sinusoidal functions of time and mechanical angle $\theta_{\mathrm{m}}$ :

$$
\mathbf{B}(\theta, t)=\sum B_{h} \cos \left(h \omega t+\phi_{h} \pm p \theta_{m}\right)
$$

where $\mathrm{B}_{\mathrm{h}}$ is magnitude of harmonic flux density, $h \omega$ and $\phi_{\mathrm{h}}$ are its angular frequency and phase angle, and $\mathrm{p}$ is pole pairs number. Besides, magnets being rarely in elongated form, the widths are often comparable to the lengths.

We should note that the assumption of homogeneous B can be valid, in any case, only when the skin effect is negligible. Therefore, we should, at first, determine the range of parameters, in which not only the skin effect can be neglected but also the $\mathrm{B}$ can be considered as homogeneous.

\section{B. Conditions for Assumption of Homogeneous Flux Density}

With both half the width and half the length of segmentation (Fig.1) less than the skin depth of interest $\delta(w / 2<\delta$ and $l / 2$ $<\delta$ ), or with only half the width less than the skin depth and the width much less than the length $(w / 2<\delta$ and $w<<l)$, we can consider the skin effect negligible in magnet segments.

Under this condition, the flux density over the width of magnet is a sinusoidal function (2). The flux, which penetrates the $\mathrm{k}^{\text {th }}$ magnet segment in Fig.1, can be estimated either by integration:

$$
\Phi=\int_{\theta_{0}}^{\theta_{0}+\Delta \theta} B(\theta, t) \cdot l \cdot r \cdot d \theta
$$

or by multiplication between an "average" flux density value at center of segment and segmentation surface area: 
$\Phi=B\left(\theta=\theta_{0}+1 / 2 \Delta \theta, t\right) \cdot l \cdot r \cdot \Delta \theta$

with $\Delta \theta=\frac{2 \pi}{3 N_{\theta} p}, \mathrm{~N}_{\theta}$ : number of pole-arc segments, and the PM covering only $2 / 3$ of one pole.

The equation (4) gives good evaluation, compared with (3), when $\mathrm{N}_{\theta}$ is greater than 2. This approximation has been verified both for unique harmonic $+6 \omega$ or $-6 \omega$, and for the composed one $(+6 \omega \wedge-6 \omega)$.

We can note that the condition to neglect skin effect is more strict than $\mathrm{N}_{\theta}>2$ for our studied machine. In other words, when the skin effect is negligible, the assumption of homogeneous flux density over magnet segment can also be established. Under this condition, we can evaluate the effect of PM segmentations on the reduction of losses.

\section{Reduction of Eddy-Current Losses by Circumferential and Axial Segmentation}

Consider a PM segmentation with width $w$ and length $l$ (Fig.1). We assume that the eddy-currents circulate in paths in rectangular form with a fixed ratio between width and length, e.g. $w / l$. Time variant flux density B induces voltage $e$ in one of these paths, 1234 (Fig.1):

$e=-\frac{d \mathbf{B}\left(\theta=\alpha_{k}, t\right) \cdot S}{d t}=-4 \frac{l}{w} x^{2} \frac{d \mathbf{B}\left(\theta=\alpha_{k}, t\right)}{d t}$

where $\mathrm{S}$ being area closed by the path 1234 is given by $2 \mathrm{x} * 2 \mathrm{y}=4 l / w x^{2}$ with $\mathrm{x} / \mathrm{y}=w / l$, the flux density $\mathrm{B}$ is vector sum of all its harmonic components (2) and $\alpha_{k}$ is centre of the $k^{\text {th }}$ segment. Resistance $\mathrm{R}$ of this path is given by:

$R=\rho\left(\frac{4 x}{h d y}+\frac{4 y}{h d x}\right)=\rho \frac{4 x\left(\frac{w}{l}+\frac{l}{w}\right)}{h d x}$

where $\mathrm{h}$ is the radial height of magnets and $\mathrm{dy}=l / w^{*} \mathrm{dx}$. Thus, differential power losses are:

$d P_{\text {ind }}=\frac{e^{2}}{R}=\frac{4}{\rho} \cdot \frac{l}{w} \cdot \frac{1}{1+(w / l)^{2}} h x^{3}\left(\frac{d \mathbf{B}\left(\theta=\alpha_{k}, t\right)}{d t}\right)^{2} d x$

The instantaneous eddy-current losses in magnet segment can be obtained by integrating $\mathrm{x}$ from 0 to $w / 2$ :

$P_{\text {ind } \_k}=\int_{0}^{w / 2} d P_{\text {ind }}=\frac{V O L}{16 \rho} \cdot \frac{w^{2}}{1+(w / l)^{2}} \cdot\left(\frac{d \mathbf{B}\left(\theta=\alpha_{k}, t\right)}{d t}\right)^{2}$

where VOL being volume of the magnet segment is given by $w^{*} l * h$.

In order to obtain the average losses in one period, we must calculate the rms value of deviation of the flux density B.
When calculating the rms value of a sum of several sinusoidal functions with different frequencies:

$$
\begin{aligned}
f=f_{6}+f_{12}=\underbrace{A \sin \left(6 \omega t+\theta_{A}\right)}_{f_{6 A}}+\underbrace{B \sin \left(6 \omega t+\theta_{B}\right)}_{f_{6 B}}+ \\
\underbrace{D \sin \left(12 \omega t+\theta_{D}\right)}_{f_{12 C} \sin \left(12 \omega t+\theta_{C}\right)}
\end{aligned}
$$

we obtain

$$
\begin{aligned}
f_{r m s} & =\sqrt{\begin{array}{l}
\left(\frac{1}{2} A^{2}+\frac{1}{2} B^{2}+A B \cos \left(\theta_{A}-\theta_{B}\right)\right)+ \\
\left(\frac{1}{2} C^{2}+\frac{1}{2} D^{2}+C D \cos \left(\theta_{C}-\theta_{D}\right)\right)
\end{array}} \\
& =\sqrt{f_{6_{-} r m s}^{2}+f_{12_{-} r m s}^{2}}
\end{aligned}
$$

It means that we can separately calculate the rms value of the components with different frequencies (e.g. $\mathrm{f}_{6}$ and $\mathrm{f}_{12}$ ) and then, get the integral rms value by root square summation. However, this does not work for the "relevant harmonic" components rotating with the same frequency in opposite direction. The integral rms value of $f_{6}$, composed of two components with the same frequency (e.g. $f_{6 A}$ and $f_{6 B}$, the indices $\mathrm{A}$ and $\mathrm{B}$ for opposite direction of rotation), dependent upon their phase difference (e.g. $A B \cos \left(\theta_{A}-\theta_{B}\right)$ ), can't be obtained simply from their own individual rms values (e.g. $1 / 2 \mathrm{~A}^{2}$ et $1 / 2 \mathrm{~B}^{2}$ ).

In such cases, we should calculate the vector sum of the two flux density components with the same frequency, and then evaluate losses due to the composed flux density, as has been shown in [6]. Taking the harmonics $6 \omega$ for example, the composed flux density is:

$$
\begin{aligned}
& B_{+6 \wedge-6}\left(\theta_{m}=\alpha_{k}, t\right)= \\
& \quad B_{+6} \cos \left(6 \omega t+\varphi_{+6}-p \alpha_{k}\right)+B_{-6} \cos \left(6 \omega t+\varphi_{-6}+p \alpha_{k}\right)
\end{aligned}
$$

where $B_{+6}$ is flux density magnitude and $\phi_{+6}$ is phase angle of the $+6 \omega$ component, $B_{-6}$ and $\phi_{-6}$ are those of the $-6 \omega$ component.

The time average eddy-current losses in single magnet segment due to the relevant time harmonics become:

$$
P_{\text {ind }_{-} k_{-} 6}=\frac{V O L}{16 \rho} \cdot \frac{w^{2}}{1+(w / l)^{2}} \cdot\left[\begin{array}{l}
\frac{1}{2}\left(B_{-6}-B_{+6}\right)^{2}(6 \omega)^{2}+ \\
2 B_{+6} B_{-6}(6 \omega)^{2} \cdot \\
\cos ^{2}\left(\frac{\varphi_{+6}-\varphi_{-6}}{2}-p \alpha_{k}\right)
\end{array}\right]
$$

Thus, the time average eddy-current losses in magnets of one pole are: 


$$
\begin{aligned}
& P_{\text {total } \_6}=\frac{N_{\theta} \cdot N_{Z} \cdot V O L}{16 \rho} \cdot \frac{w^{2}}{1+(w / l)^{2}} \cdot \\
& {\left[\begin{array}{c}
2 B_{+6} B_{-6}(6 \omega)^{2} \cdot \frac{1}{N_{\theta}} \cdot \underbrace{\sum_{k=1}^{N_{\theta}} \cos ^{2}\left(\frac{\varphi_{+6}-\varphi_{-6}}{2}-p \alpha_{k}\right)}_{F}+] \\
\frac{1}{2}\left(B_{-6}-B_{+6}\right)^{2}(6 \omega)^{2}
\end{array}\right]}
\end{aligned}
$$

with $w=\mathrm{W} / \mathrm{N}_{\theta}$ and $l=\mathrm{L} / \mathrm{N}_{\mathrm{Z}}, \mathrm{W}$ : total pole-arc width of magnets on one pole, $\mathrm{L}$ : total axial length of magnets on one pole, $\mathrm{N}_{\theta}$ : number of pole-arc segments, $\mathrm{N}_{\mathrm{Z}}$ : number of axial segments

We have proved that the function $\mathrm{F} / \mathrm{N}_{\theta}$ in (13) is quasiindependent of $\mathrm{N}_{\theta}$ for $\mathrm{N}_{\theta}>2$ (Fig.2) but it depends on the phase difference between two relevant harmonics $\left(\varphi_{+6}-\varphi_{-6}\right) / 2$.

The eddy-current losses are proportional to $\frac{w^{2}}{1+(w / l)^{2}}$.

Comparing to the traditional analytical model (1) with only $w$ taken into account, we consider magnets in any rectangular form, with both dimensions $w$ and $l$ explicitly engaged (Fig.1). In the contrary to the equation proposed in [5], this relationship is simple and clearly represents the effect of circumferential and axial segmentation on the eddy-current losses.

In short, to exploit equation (13) for eddy-current losses prediction, we should:

(a) determine skin depth $\delta$

(b) determine the parameter range where skin effect is negligible : $(w / 2<\delta$ and $l / 2<\delta)$ or $(w / 2<\delta$ and $w<<l)$

(c) in these cases, apply equation (13) to evaluate eddycurrent loss reduction due to PM segmentations

Whenever the skin effect is operating, the analytical model (1) and (13) are inappropriate. The skin effect leads to nonhomogeneous flux density and lowers the total flux passing through the magnets. We will discuss in next chapter influence of the skin effect on the efficiency of PM segmentation to reduce the eddy-current losses.

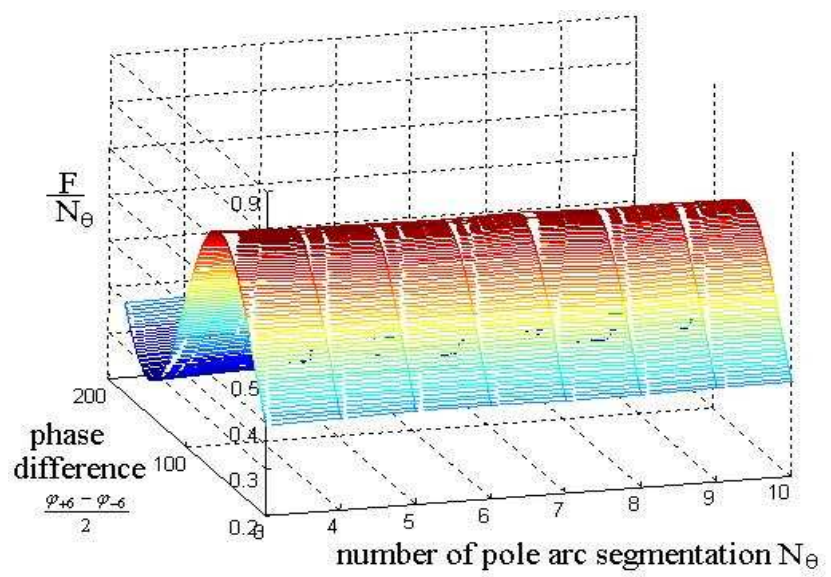

Fig. 2. Function $F / N_{\theta}$ in the equation (13) is independent of $N_{\theta}$, which implies that the term between parentheses is also independent of $\mathrm{N}_{\theta}$.

\section{EDDY-CURRENT LOSSES IN CASES OF THE SKIN EFFECT OPERATING}

\section{A. High Frequency Eddy-Current Losses}

A useful formula (14) for high frequency losses $(10 \mathrm{kHz}$ with $\delta=0.078 \mathrm{~mm}$, for lamination thick $0.356 \mathrm{~mm}$ ) has been reported in [8] to evaluate the eddy-current losses in laminated steel when skin depth $\delta$ is much less than (actually at least 5 times smaller) the material thickness.

$$
P / S=\frac{1}{2}\left|H_{\tan }\right|^{2} R_{S}
$$

where $\mathrm{H}_{\text {tan }}$ is the peak tangential incident magnetic intensity and $R_{\mathrm{s}}$ is the surface impedance in ohm per square given by $1 / \delta \sigma$. It shows that specific eddy-current losses per tangential surface are independent of material dimensions.

We apply this formula for losses in magnets, $\mathrm{S}$ being given by $2 *(l+w) * \mathrm{~h}$. Thus, the eddy-current losses per unite volume are:

$$
P_{v}=\frac{P}{V O L}=\frac{P}{S / 2 \cdot w l /(w+l)} \propto\left(\frac{1}{l}+\frac{1}{w}\right)
$$

When diminishing the width or/and the length of magnet segments, the eddy-current losses increase. These are conditions of unexpected deviation from the classical rule of segmentation which states that the eddy-current losses in magnet diminish as we proceed with its division. Actually, they can rise. We call it the "anomaly of segmentation".

\section{B. Any Frequency Eddy-Current Losses}

A general formula has been presented in [10] for any frequency, but only for conductor in elongated form with $w<<l$ :

$P_{V}=\sqrt{\frac{\rho \pi f}{\mu^{3}}} \frac{1}{w} B_{s}^{2} \frac{\operatorname{sh} w / \delta-\sin w / \delta}{\operatorname{ch} w / \delta+\cos w / \delta}$

where $B_{s}$ is peak of surface flux density and $\mu$ is magnet permeability.

According to this equation, when diminishing the magnet's width $w$, the total eddy-current loss will increase at first and then decrease. This confirms both "anomaly of segmentation" and the classical reduction of the losses with magnet segmentation.

Using this equation, we can detect maximum eddy-current losses with respect to PM circumferential segmentation, i.e. "peak of eddy current losses". This peak separates two situations: (i) segmentation of PM increases the eddy-current losses; (ii) segmentation of PM reduces the eddy-current losses. However, under the condition $w<<l$, only the circumferential segmentation effect can be taken into account. 


\section{Peak of Eddy-Current Losses versus Circumferential and Axial Segmentations}

The aim of this section is to find out a simple criterion to detect the peak of eddy current losses with respect to circumferential and axial segmentations. We begin with analyzing distribution of flux in conduction area with skin effect.

The flux density in the magnet's volume (Fig.1) can be represented as a function of the pole-arc coordination $\mathrm{x}$ with Bs being the flux density on the magnet's surface:

$$
B(x)=B_{s} \frac{\cos ((1+j) x / \delta)}{\cos ((1+j) d / 2 \delta)}
$$

The flux passing through the surface $\left(w^{*}\right)$ of the conducting area is given by:

$$
\Phi=\int_{0}^{w / 2} B(x) \cdot l \cdot d x=\left(w \cdot l \cdot B_{s}\right) \cdot \frac{2 \delta}{w} \frac{1}{1+j} \tan \left((1+j) \frac{w}{2 \delta}\right)
$$

With the ratio $w / 2 \delta$ much less than 1 (skin effect negligible), we can consider the flux density within the magnet's volume as homogeneous and equal to the surface flux density Bs. On the contrary, with $w / 2 \delta$ higher than 1 (skin effect operating), the skin effect prevents homogeneous penetration of magnetic flux into the conduction area. The flux density is higher in proximity of two edges at $\mathrm{x}=w / 2$ and $\mathrm{x}=-w / 2$ than in the center at $\mathrm{x}=0$ (Fig.3.a). For $w / 2 \delta \geq 2$, we can approximate the total flux by:

$|\Phi| \approx\left(w \cdot l \cdot B_{s}\right) \cdot \frac{2 \delta}{w} \frac{1}{\sqrt{2}}=2\left(\frac{\delta}{\sqrt{2}} \cdot l \cdot B_{s}\right)$

since $\tan (1+j) w / 2 \delta \approx j$.

In other words, when the skin effect is important, we can model the non-homogeneous flux density by two bands of homogeneous flux density with thickness $\delta / \sqrt{2}$ and magnitude B $_{\mathrm{s}}$ (Fig.3.a). We call them "dissipation bands" because most of the eddy-current losses are in these two areas.

With the number of the pole-arc segments $\mathrm{N}_{\theta}=2$, if half of the segment width is still much greater than $\delta$, number of dissipation bands will become double, as well as the eddycurrent losses (Fig.3.b).

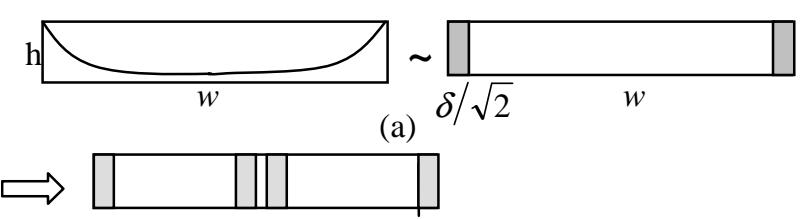

(b)

Fig. 3. (a). The skin effect leads to non-homogeneous flux distribution in magnets. This can be modeled by two bands with homogeneous flux. (b). Segmentation doubles number of bands, as well as eddy-current losses.
Considering model of Fig.3, we can predict the existence of a simple criterion to estimate location of the peak of eddycurrent losses with respect to circumferential and axial segmentations of PM:

$\frac{w}{2 \delta} \approx 2$ and $\frac{l}{2 \delta} \approx 2$

This is the generic condition to assure "absolute nonhomogeneity" of the flux density, which permits to model the skin effect by two bands of homogeneous flux density. In other words, it's the limit to authorize the approximation of (19).

\section{FinITE ELEMENT SIMULATION}

3D harmonic finite element (FE) analysis has been used to validate the proposed prediction technique. Only one of the six pole-pairs in studied PM synchronous machine has been modeled, with periodic boundary conditions in $\theta$ direction (Fig.4). In $\mathrm{Z}$ direction, with aid of periodic boundary conditions, we can model part of machine corresponding to one magnet segment length, (e.g. $25 \mathrm{~mm}$ when number of axial segments $\mathrm{N}_{\mathrm{z}}=10$ ), instead of the entire length of machine (250mm). The PM covers only $2 / 3$ of a pole pair arc.

Since the analytical method does not consider losses resulting from variation of magnet working point due to stator slot opening, its results can only be compared with those from FE calculation with the magnets unmagnetized and modeled as simple conductor. The ignored contribution however can frequently be considered as negligible.

To overcome time consuming problem of 3D time-stepping FE analysis, the locked rotor technique is applied. We determine the harmonic frequency of stator currents so that harmonic MMF rotates around the locked rotor at correct relative angular velocity, e.g. $6 \omega(1800 \mathrm{~Hz}), 12 \omega(3600 \mathrm{~Hz})$, $18 \omega(5400 \mathrm{~Hz}) \ldots$ etc.

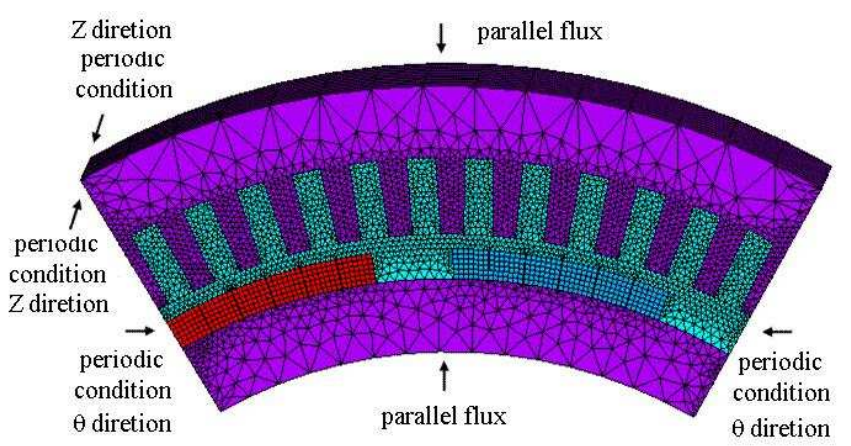

Fig. 4. Finite element model of one pole-pair of PM synchronous machine, with 6 PM segments in circumferential direction and 10 in axial direction. The dimension in $\mathrm{Z}$ direction of this model corresponds to $1 / 10$ of the entire machine length, i.e. $250 / 10=25 \mathrm{~mm}$. 


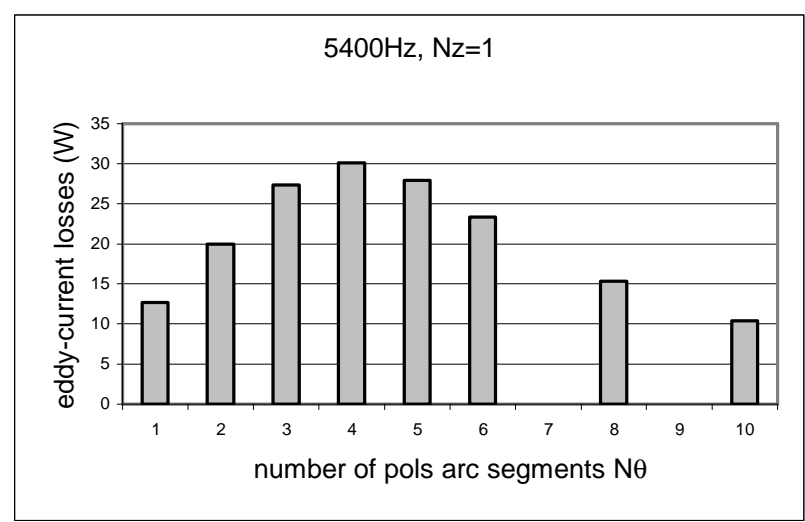

Fig. 5. Variation of the eddy-current losses with respect to number of pole arc magnet segments at $\mathrm{f}=5400 \mathrm{~Hz}$ and with only one segment in $\mathrm{Z}$ direction.

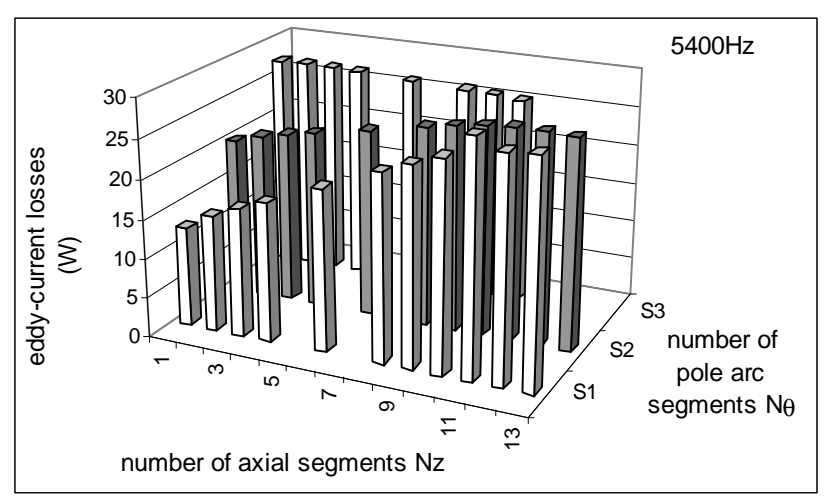

Fig. 6. Variation of the eddy-current losses with respect to number of pole arc and of axial magnet segments, at $\mathrm{f}=5400 \mathrm{~Hz}$

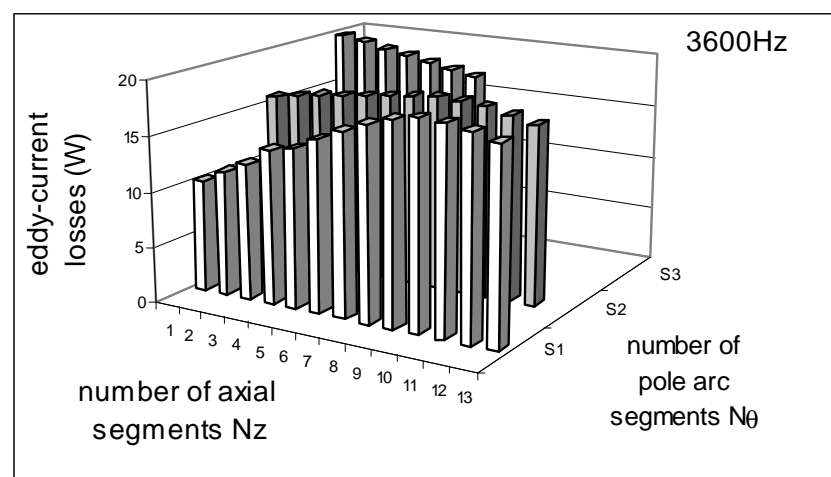

Fig. 7. Variation of the eddy-current losses with respect to number of pole arc and of axial magnet segments, at $\mathrm{f}=3600 \mathrm{~Hz}$

Three groups of relevant harmonics, inducing eddy-currents at 1800,3600 and $5400 \mathrm{~Hz}$, are discussed. At $5400 \mathrm{~Hz}$, the skin depth $\delta$ being $6.2 \mathrm{~mm}$, the peak of losses versus the pole-arc segmentations, by keeping $\mathrm{Nz}=1$, locates at $\mathrm{N}_{\theta}=4$ where $w / 2 \delta=1.4$ (Fig.5). Circumferential segmentations rise at first and then reduce the eddy-current losses.

Similar phenomena can be found with axial segmentations. The peak of losses versus the axial segmentations, by keeping $\mathrm{N} \theta=1$, locates at $\mathrm{Nz}=11$ where $l / 2 \delta=1.8$ (Fig.6).

Within this range of parameters, $\mathrm{N}_{\theta} \leq 4$ and $\mathrm{Nz} \leq 11$ at $5400 \mathrm{~Hz}$, circumferential and axial segmentations can not lead to the expected reduction of losses in magnets.

At lower frequencies, the skin effect being less remarkable, the range of parameters, within which the anomaly of PM segmentation takes place, is reduced. For example, at $3600 \mathrm{~Hz}$ (Fig.7) the range is: $\mathrm{N}_{\theta} \leq 3(w / 2 \delta=1.5)$ and $\mathrm{Nz} \leq 10(l / 2 \delta=1.6)$ and at $1800 \mathrm{~Hz}$ (Fig.8), it is: $\mathrm{N}_{\theta} \leq 2(w / 2 \delta=1.6)$ and $\mathrm{Nz} \leq 7$ $(1 / 2 \delta=1.7)$.

In Fig.8, we can note that the segmentation, in direction of magnet's minimum dimension over the surface perpendicular to flux direction, i.e. circumferential (pole arc) direction, is more efficient than the axial one.

In cases when skin effect can be neglected, e.g. at $1800 \mathrm{~Hz}$ for $\mathrm{N}_{\theta}=(4,5,6,10)$ and $\mathrm{N}_{\mathrm{Z}}=1$, or for $\mathrm{N}_{\theta}=10$ and $\mathrm{N}_{\mathrm{Z}}=(1,3,6)$, we can compare prediction of the effect of circumferential and axial segments by the ratio $\frac{w^{2}}{1+(w / l)^{2}}$ in (13) with results by

FE simulation (Table I). Taking the case with four pole-arc segments and one axial segment as base value of the eddycurrent losses, the effect of segmentations for reduction of losses is represented by a ratio less than 1 .

\section{CONCLUSION}

In cases when the skin effect is negligible any circumferential or axial segmentations of magnets always leads to reduction of eddy-current losses. Otherwise we should count with enhancement of the eddy-current dissipation for certain schemes of magnets' division, followed by classical reduction of the eddy-current losses when proceeding with further subdivision.

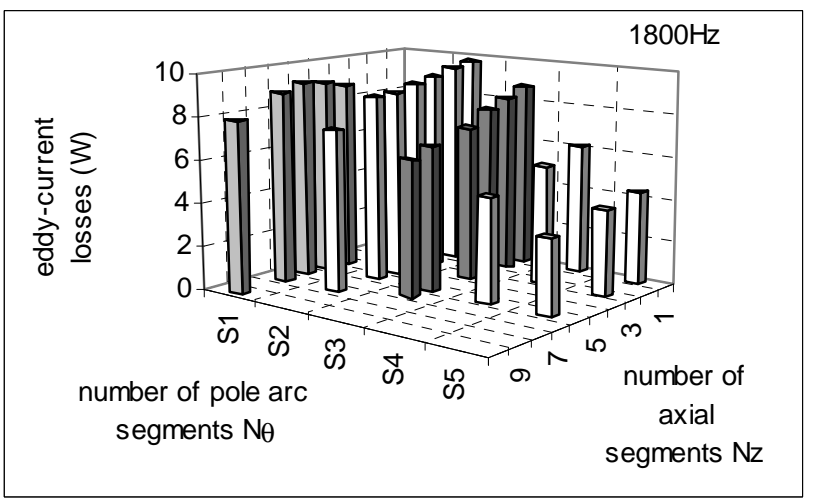

Fig. 8. Variation of the eddy-current losses with respect to number of pole arc and of axial magnet segments, at $\mathrm{f}=1800 \mathrm{~Hz}$

TABLE I

EFFECT OF CIRCUMFERENTIAL AND AXIAL SEGMENTAIONS

\begin{tabular}{ccc}
\hline \hline $\mathrm{N}_{\theta} * \mathrm{~N}_{\mathrm{Z}}$ & $\begin{array}{c}\mathrm{P} / \mathrm{P}_{4 * 1} \\
\mathrm{By} \mathrm{EF}\end{array}$ & $\begin{array}{c}\mathrm{P} / \mathrm{P}_{4} *_{1} \\
\mathrm{By}(13)\end{array}$ \\
\hline $4 * 1$ & 1 & 1 \\
$5 * 1$ & 0.7 & 0.64 \\
$6 * 1$ & 0.51 & 0.45 \\
$10 * 1$ & 0.197 & 0.161 \\
$10 * 3$ & 0.188 & 0.160 \\
$10 * 6$ & 0.179 & 0.156 \\
\hline
\end{tabular}


A simple criterion has been proposed to detect the geometries of PM segments corresponding to the point of maximum eddy-current losses.

The analysis concerning one frequency on the rotor side, it can be applied to one pair of stator harmonics which generate this frequency - e.g. $5^{\text {th }}$ and $7^{\text {th }}$ generating the $6^{\text {th }}$ in the rotor's magnets. Whenever higher harmonics are also relevant, we can easily conclude on a compromise on actual subdivision rule.

An efficient way of the finite element verification is the locked-rotor method with its simpler modeling and less computational strains comparing to the time-stepping procedures.

\section{REFERENCES}

[1] H. Polinder and M.J. Hoeijmakers, "Eddy-current losses in the permanent magnets of a PM machine", EMD97, Sep. 1997, pp.138-142

[2] Kais Altallah et al, "Rotor loss in Permanent-magnet brushless AC machines", IEEE Trans. on Industry Applications, vo. 36, no. 6, Nov. 2000, pp. 1612-1617

[3] Hiroaki Toda et al, "Rotor eddy-current loss in permanent magnet brushless machines", IEEE Trans. on Magnetics, vo. 40, no. 4, July 2004, pp.2104-2106

[4] Jason D. Ede et al, "Effect of Axial segmentation of permanent magnets on rotor loss in modular permanent-magnet brushless machines", IEEE Trans. on Industry Applications, Vo. 43, no.5, Sep. 2007, pp. 12071213

[5] K. Yamazaki and A. Abe, "Loss Investigation of interior permanentmagnet motors considering carried harmonics and magnet eddy currents", IEEE Trans. on Industry Applications, Vo. 45, no.2, March/April 2009, pp. 659-665

[6] R. Kaczmarek, W-Y Huang, and J-C Vannier, "Magnetic Losses Evaluation in PM SM Drive with Non Sinusoidal Supply by Finite Elements Analysis and Superposition Principle", SDEMPED 2007, Cracovie

[7] Leander W. Matsch, J. Derald Morgan, "Electromagnetic and electromechanical machines", John Wiley \& Sons, $3^{\text {rd }}$ edition

[8] John R. Brauer et al, "Laminated steel eddy-current loss versus frequency computed using finite elements", IEEE Trans. on Industry Applications, vo. 36, no.4, August 2000, pp1132-1137

[9] James L. Kirtley Jr., "Eddy Currents, Surface Impedance and loss Mechanisms", Class Notes of Massachusetts Institute of Technology Department of Electrical Engineering and Computer Science, September 5,2005

[10] G. Bertotti, «Hysteresis in magnetism- For physicists, materials scientists, and engineers », Academic Press, 1998

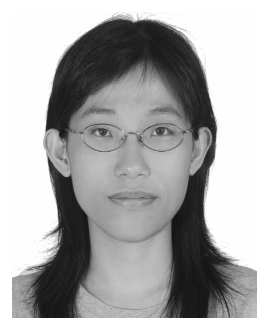

Wan-Ying HUANG has graduated from Tsing Hua University in Taiwan with Master Degree in 2002 and in 2006 has got her PHD in Ecole Supérieure d'Electricité SUPELEC and University Paris XI in France. She is now on a post doctoral research program in SUPELEC, working on stability items in electrical systems and optimal design of electric machines.

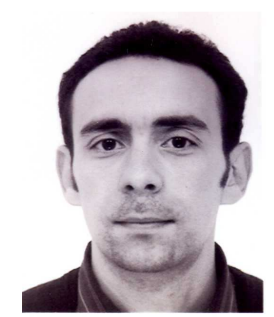

Adel BETTAYEB received the Ingénieur d'Etat Dergree in electrical power engineering from the U.S.T.H.B University, Algiers, Algeria, in 2004 and the Master Degree from the University of Paul Sabatier, Toulouse, F.R.., in 2007. In December $2007 \mathrm{He}$ is currently a PhD student in Ecole Supérieure d'Electricité SUPELEC (France). His research interests are on the rotor iron losses in synchronous machines.

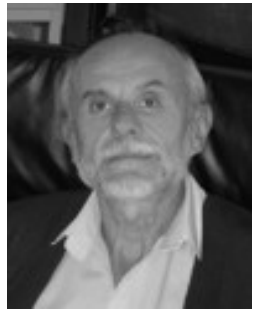

Robert KACZMAREK is professor in Ecole Supérieure d'Electricité (Supelec) in France. His research interest is with power networks (fault detection, network protection, and parameters identification), magnetism (domain observation, loss prediction and measurements in soft and hard magnetic materials) and design of electric machines.

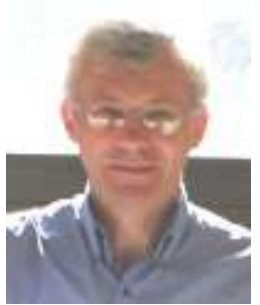

Jean-Claude VANNIER is professor and Head of Department of Electrical Machines and Power Systems in the Ecole Supérieure d'Electricité (Supelec) in France. His research interest is with energy conversion systems (motors, actuators, generators) and concerns the modeling, the design and the optimization of these equipments for specific applications. 